\title{
A dynamic model of the pregnant myometrial fasciculus
}

\author{
R. Miftahof ${ }^{1} \&$ N. Akhmadeev ${ }^{2}$ \\ ${ }^{I}$ Arabian Gulf University, Manama, Kingdom of Bahrain \\ ${ }^{2}$ Kazan Medical University, Russia
}

\begin{abstract}
A mathematical model of the pregnant myometrial fasciculus is proposed. It is based on real anatomical and physiological data of its structure and function. The model reproduces electromechanical wave phenomena in the myometrium under normal physiological conditions and after application of different classes of pharmacologically active compounds.

Keywords: human myometrium, fasciculus, electromechanical waves.
\end{abstract}

\section{Introduction}

In recent years the biomedical research on reproduction has been focused mainly on molecular, neuroendocrine and pharmacological aspects of uterine activity. A relatively small amount of work has been dedicated to modeling of the uterus per $s e$. Various approaches have been developed for uterine mechanics modeling during parturition. The majority of them rely on assumptions of geometrical and physical linearity $[1,2]$. Results of numerical simulations obtained for different values of parameters and constants resembled patterns of spontaneous contractility observed during normal labor and reproduced voltage-clamp traces recorded experimentally on pregnant rats and human non-pregnant myometrial cells. Although the existing models of the myometrium and the gravid human uterus are based on the accurate application of general principles of solid mechanics and incorporate some morphological data on the structure and function of the organ, these models remain of limited biomedical value. New integrative, biologically plausible models are needed to answer urgent questions related to clinical problems of labor and delivery. 
The aims of this study were twofold: i) to formulate a biomechanical model of the pregnant human myometrial fasciculus, and ii) to study numerically the biological phenomena that underlie processes of electromechanical activity in it.

\section{Model formulation}

Consider a one-dimensional model of the dynamics of fasciculus (myofiber) - the functional unit of the human uterus. Let a fasciculus be embedded in the extracellular matrix of connective tissue. Our developments of a biomechanical model will be based on the following assumptions which are consistent with several lines of experimental evidence $[3,4]$.

i) Smooth muscle cells in the fasciculus are connected by tight junctions to form a homogenous electromechanical continuum.

ii) The myofiber possesses nonlinear viscoelastic properties; the mechanics of inactive smooth muscle cells, collagen, elastin fibers and the extracellular matrix define the "passive", $T^{p}\left(\lambda, c_{i}\right)$, and intracellular contractile proteins describe the "active" component, $T^{a}\left(\lambda, Z_{m n}^{(*)},\left[\mathrm{Ca}_{i}^{2+}\right], c_{i}\right)$, of the total force, $T^{t}$

$$
T^{t}=T^{p}\left(\lambda, c_{i}\right)+T^{a}\left(\lambda, Z_{m n}^{(*)},\left[\mathrm{Ca}_{i}^{2+}\right], c_{i}\right),
$$

where $\lambda$ is the stretch ratio, $c_{i}$ are empirical material constants, $Z_{m n}^{(*)}$ is the "biofactor", and $\left[\mathrm{Ca}_{i}^{2+}\right]$ is the concentration of free cytosolic calcium.

iii) Contractions of the fiber are isometric; deformations are finite.

iv) Myogenic electrical events are a result of activity of an intrinsic autonomous oscillator; its function is defined by the fast (T-type) and slow (L-type) inward $\mathrm{Ca}^{2+}, \mathrm{BK}_{\mathrm{Ca}}$, voltage dependent $\mathrm{K}_{\mathrm{v} 1}{ }^{+}$and leak $\mathrm{Cl}^{-}$currents.

v) Each oscillator is in the silent state; the transformation to a firing state is a result of depolarization and/or stretch deformation of the cell that alters the conductance for L- and T-type $\mathrm{Ca}^{2+}$ channels, while the stretch affects permeability of L-type channels.

vi) The myofiber possesses cable electrical properties; propagation of the wave of depolarization is a result of combined activity of the $\mathrm{Na}^{+}, \mathrm{K}_{\mathrm{v} 2}{ }^{+}$, and leak $\mathrm{Cl}^{-}$ion currents.

vii) A smooth muscle cell or a group of cells within the fasciculus have intrinsic pacemaker properties; the transformation from a silent to a bursting state can occur spontaneously and is a result of "alterations" in electrical properties; additionally, an a priori defined "pacemaker" provides an excitation to the fiber. 
Let the fasciculus of a length $L$ be referred to a local Lagrange coordinate system $\alpha$. Its equation of motion is given by

$$
\rho \frac{\partial v}{\partial t}=\frac{\partial}{\partial \alpha} T^{t}, \quad(0 \leq \alpha \leq L)
$$

where $\rho$ is density, $v$ is the velocity, and the meaning of other parameters are as described above. Following the working assumption ii), the total force $T$ can be decomposed as

$$
T^{t}=k_{v} \frac{\partial(\lambda-1)}{\partial t}+T^{a}\left(\lambda, Z_{m n}^{(*)},\left[\mathrm{Ca}_{i}^{2+}\right], c_{i}\right)+T^{p}\left(\lambda, c_{i}\right),
$$

where the viscoelastic term has been added to Eq. (1). Here $k_{v}$ is viscosity. Substituting the above into Eq. (3) we obtain

$$
\rho \frac{\partial v}{\partial t}=\frac{\partial}{\partial \alpha}\left(k_{v} \frac{\partial(\lambda-1)}{\partial t}+T^{a}\left(\lambda, Z_{m n}^{(*)},\left[\mathrm{Ca}_{i}^{2+}\right], c_{i}\right)+T^{p}\left(\lambda, c_{i}\right)\right),
$$

where the force-stretch ratio relationship yields

$$
T^{p}=\left\{\begin{array}{lr}
c_{1}\left[\exp c_{2}(\lambda-1)-1\right], & \lambda>1.0 \\
0, & \text { otherwise }
\end{array}\right.
$$

and the active-force - intracellular $\mathrm{Ca}_{i}^{2+}$ relationship for the myometrium is given by

$$
T^{a}=\left\{\begin{array}{lr}
0, & {\left[\mathrm{Ca}_{i}^{2+}\right] \leq 0.1 \mu \mathrm{M}} \\
c_{3}+c_{4}\left[\mathrm{Ca}_{i}^{2+}\right]^{4}+c_{5}\left[\mathrm{Ca}_{i}^{2+}\right]^{3}+c_{6}\left[\mathrm{Ca}_{i}^{2+}\right]^{2}+c_{7}\left[\mathrm{Ca}_{i}^{2+}\right], & 0.1<\left[\mathrm{Ca}_{i}^{2+}\right] \leq 1 \mu \mathrm{M} \\
\max T^{a}, & {\left[\mathrm{Ca}_{i}^{2+}\right]>1 \mu \mathrm{M} .}
\end{array}\right.
$$

The system of equations for the oscillatory activity of the membrane potential $V$ is

$$
\lambda C_{m} \frac{d V}{d t}=-\sum_{j} \tilde{I}_{j}
$$


where, $\lambda$ is the numerical parameter, $C_{m}$ is the uterine smooth muscle cell membrane capacitance, and $\tilde{I}_{j}$ is the sum of the respective ion currents

$$
\begin{gathered}
\tilde{I}_{C a}^{f}=g_{C a}^{f} \tilde{m}_{i}^{3} \tilde{h}\left(V-V_{C a}\right), \quad \tilde{I}_{C a}^{s}=g_{C a}^{s} \tilde{x}_{C a}\left(V-V_{C a}\right), \\
\tilde{I}_{K 1}=g_{K 1} \tilde{n}^{4}\left(V-V_{K 1}\right), \quad \tilde{I}_{C a-K}=\frac{g_{C a-K}^{f}\left[\mathrm{Ca}_{i}^{2+}\right]\left(V-V_{C a}\right)}{0.5+\left[\mathrm{Ca}_{i}^{2+}\right]}, \\
\tilde{I}_{C l}=g_{C l}\left(V-V_{C l}\right) .
\end{gathered}
$$

Here $V_{C a}, V_{K 1}, V_{C l}$ are the reversal potentials, and $g_{C a}^{f}, g_{C a}^{s}, g_{K 1}, g_{C a-K}, g_{C l}$ are the maximal conductances for the ion currents, $\tilde{m}, \tilde{h}, \tilde{n}$ and $\tilde{x}_{C a}$ are dynamic variables given by

$$
\begin{gathered}
\tilde{m}_{I}=\frac{\tilde{\alpha}_{m}}{\tilde{\alpha}_{m}+\tilde{\beta}_{m}}, \quad \lambda \hbar \frac{d \tilde{h}}{d t}=\tilde{\alpha}_{h}(1-\tilde{h})-\tilde{\beta}_{h} \tilde{h}, \\
\lambda \hbar \frac{d \tilde{n}}{d t}=\tilde{\alpha}_{n}(1-\tilde{n})-\tilde{\beta}_{n} \tilde{n}, \\
\lambda \tau_{x C a} \frac{d \tilde{x}_{C a}}{d t}=\frac{1}{\exp (-0.15(V+50))}-\tilde{x}_{C a}, \\
\lambda \frac{\mathrm{d}\left[\mathrm{Ca}_{i}^{2+}\right]}{\mathrm{d} t}=\wp_{C a} \tilde{x}_{C a}\left(V_{C a}-V\right)-\left[\mathrm{Ca}_{i}^{2+}\right] .
\end{gathered}
$$

Here the activation $\tilde{\alpha}_{y}$ and deactivation $\widetilde{\beta}_{y}(y=\tilde{m}, \tilde{h}, \tilde{n})$ parameters of ion channels satisfy the empirical relations

$$
\begin{gathered}
\tilde{\alpha}_{m}=\frac{0.1(50-\tilde{V})}{\exp (5-0.1 \tilde{V})-1}, \quad \tilde{\beta}_{m}=4 \exp \frac{(25-\tilde{V})}{18}, \\
\tilde{\alpha}_{h}=0.07 \exp \frac{(25-0.1 \tilde{V})}{20}, \quad \tilde{\beta}_{h}=\frac{1}{1+\exp (5.5-0.1 \tilde{V})}, \\
\tilde{\alpha}_{n}=\frac{0.01(55-\tilde{V})}{\exp (5.5-0.1 \tilde{V})-1}, \quad \tilde{\beta}_{n}=0.125 \exp \frac{(45-\tilde{V})}{80},
\end{gathered}
$$

where $\tilde{V}=(127 V+8265) / 105, \tau_{x C a}$ is the time constant, $\wp_{C a}$ is the parameter referring to the dynamics of $\mathrm{Ca}^{2+}$ channels, $\hbar$ is a numerical constant. 
The evolution of $\mathrm{L}$ - and $\mathrm{T}$-type $\mathrm{Ca}^{2+}-$ channels depends on the wave of depolarization, $V^{s}$, and is defined by

$$
\begin{gathered}
g_{C a}^{s}(t)=[\delta(V)+(\lambda(t)-1)]\left(\max g_{C a}^{s}\right), \\
g_{C a}^{f}(t)=(\lambda(t)-1) g_{C a}^{f},
\end{gathered}
$$

where

$$
\lambda(t) \geq 1.0, \quad \delta(V)=\left\{\begin{array}{lc}
1, & \text { for } V \geq V_{p}^{s} \\
0, & \text { otherwise }
\end{array} .\right.
$$

Here $V_{p}^{s}$ is the threshold value for $V^{s}$.

The propagation of the wave of excitation $V^{s}$ is described by

$$
C_{m} \frac{\mathrm{d} V^{s}}{\mathrm{~d} t}=\frac{d_{m}}{R_{s}} \frac{\partial}{\partial \alpha}\left(\lambda(\alpha) \frac{\partial V^{s}}{\partial \alpha}\right)-\left(I_{N a}+I_{K 2}+I_{C l}\right),
$$

where $d_{m}$ is the diameter, $R_{s}$ is the specific resistance of the fasciculus, and

$$
\begin{gathered}
I_{N a}=g_{N a} \hat{m}^{3} \hat{h}\left(V^{s}-V_{N a}\right) \\
I_{K 2}=g_{K 2} \hat{n}^{4}\left(V^{s}-V_{K 2}\right) \\
I_{C l}=g_{C l}\left(V^{s}-V_{C l}\right) .
\end{gathered}
$$

Here $g_{\mathrm{Na}}, g_{K 2}, g_{C l}$ are the maximal conductances, and $V_{N a}, V_{K 2}, V_{C l}$ are the reversal potentials of $\mathrm{Na}^{+}, \mathrm{K}_{\mathrm{v} 2}{ }^{+}$, and $\mathrm{Cl}^{-}$membrane currents, respectively. The dynamics of the variables $\hat{m}, \hat{h}, \hat{n}$ are described by

$$
\frac{\mathrm{d} \hat{m}}{\mathrm{~d} t}=\hat{\alpha}_{m}(1-\hat{m})-\hat{\beta}_{m} \hat{m}, \frac{\mathrm{d} \hat{h}}{\mathrm{~d} t}=\hat{\alpha}_{h}(1-\hat{h})-\hat{\beta}_{h} \hat{h}_{,} \frac{\mathrm{d} \hat{n}}{\mathrm{~d} t}=\hat{\alpha}_{n}(1-\hat{n})-\hat{\beta}_{n} \hat{n}
$$

with the activation $\hat{\alpha}_{y}$ and deactivation $\hat{\beta}_{y}(y=\hat{m}, \hat{h}, \hat{n})$ parameters given by

$$
\begin{aligned}
& \hat{\alpha}_{m}=\frac{0.005\left(V^{s}-V_{m}\right)}{\exp 0.1\left(V^{s}-V_{m}\right)-1}, \hat{\beta}_{m}=0.2 \exp \frac{\left(V^{s}+V_{m}\right)}{38}, \\
& \hat{\alpha}_{h}=0.014 \exp \frac{-\left(V_{h}+V^{s}\right)}{20}, \hat{\beta}_{h}=\frac{0.2}{1+\exp 0.2\left(V_{h}-V^{s}\right)},
\end{aligned}
$$




$$
\hat{\alpha}_{n}=\frac{0.006\left(V^{s}-V_{n}\right)}{\exp 0.1\left(V^{s}-V_{n}\right)-1}, \quad \hat{\beta}_{n}=0.75 \exp \left(V_{n}-V^{s}\right) .
$$

Here $V_{m}, V_{h}, V_{n}$ are the reversal potentials for activation and inactivation of $\mathrm{Na}^{+}$and $\mathrm{K}_{\mathrm{v} 2}{ }^{+}$ion currents of the myometrium.

In the following numerical experiments we assume that at the initial moment of time the functional unit is in unexcitable state

$$
\begin{gathered}
V^{s}(\alpha, 0)=0, v(\alpha, 0)=0,\left[\mathrm{Ca}_{i}^{2+}\right]=\left[\mathrm{Ca}_{i}{ }^{2+}\right], \\
\hat{m}=\hat{m}_{\infty}, \hat{h}=\hat{h}_{\infty}, \hat{n}=\hat{n}_{\infty}, \tilde{h}=\tilde{h}_{\infty}, \tilde{n}=\tilde{n}_{\infty}, \tilde{x}_{C a}=\tilde{x}_{C a}^{\infty} .
\end{gathered}
$$

It is activated by a series of discharges of action potentials by an intrinsic pace-maker cell

$$
V^{s}(0, t)=\left\{\begin{array}{ll}
0 & \\
V^{s}, & 0<t<t^{d} \\
0, & t \geq t^{d}
\end{array}, \quad V^{s}(0, t)=V(t),\right.
$$

The ends of the myofiber are clamped and remain unexcitable throughout

$$
V^{s}(0, t)=V^{s}(L, t)=0, \quad v(0, t)=v(L, t)=0 .
$$

Eqs. (4)-(15), initial and boundary conditions (16) -(18) constitute the mathematical formulation of the model of the electromechanical activity of the myometrial fasciculus. It describes:

i) self oscillatory behavior and/or myoelectrical activity induced by discharges of a "pacemaker" cell;

ii) generation and propagation of the wave of depolarization along the myofiber;

iii) coupling of spatially distributed oscillators;

iv) generation of action potentials;

v) dynamics of the cytosolic $\mathrm{Ca}^{2+}$ transients;

vi) active and passive force generation;

vii) deformation of the fasciculus and the following excitation of the cell membrane with contractions.

The governing system of equations was solved numerically using $A B S$ Technologies@ software.

\section{Results of numerical simulations}

\subsection{Physiological condition}

The resting membrane potential of the fasciculus is $V^{r}=-59 \mathrm{mV}$. Continuous fluctuations at low rate and amplitudes of the $\mathrm{L}$-type $-0.08 \mathrm{nA}, \mathrm{T}$-type $\mathrm{Ca}^{2+}$ $0.48 \mathrm{nA}$, respectively, an outward $\mathrm{K}^{+}-0.03 \mathrm{nA}$, the $\mathrm{BK}_{\mathrm{Ca}}-0.62 \mathrm{nA}$, and the small chloride $-0.04 \mathrm{nA}$ currents result in oscillations of the membrane potential 
$V$ known as slow waves. Their frequency, $v=0.02 \mathrm{~Hz}$, and the amplitude, $V=27$ $\mathrm{mV}$, remain constant. The maximum rate of depolarization is calculated $9 \mathrm{mV} / \mathrm{s}$ and of repolarization $-7.5 \mathrm{mV} / \mathrm{s}$.

The slow wave induces the flux of $\mathrm{Ca}^{2+}$ ions inside the cell at a rate of 0.057 $\mu \mathrm{M} / \mathrm{s}$. There is a $20 \mathrm{sec}$ time delay in the intracellular calcium transients as compared to the wave of depolarization. Free cytosolic calcium at $\max \left[\mathrm{Ca}^{2+}\right]=$ $0.44 \mu \mathrm{M}$ activates the contractile protein system with the production of spontaneous contractions, $T^{a}=13.6 \mathrm{mN} / \mathrm{cm}$ (Fig. 1). They follow in phase and time the dynamics of calcium oscillations and are normally preceded by slow waves.

High frequency discharges of an intrinsic pacemaker initiate high magnitude ion currents: $\tilde{I}_{C a}^{s}=0.4, \tilde{I}_{C a}^{f}=0.51, \tilde{I}_{K 1}=0.2, \tilde{I}_{C a-K}=1.0$, and $\tilde{I}_{C l}=0.5(\mathrm{nA})$, and the generation of action potentials of amplitudes $38 \div 45 \mathrm{mV}$ at a frequency of $2.7 \mathrm{~Hz}$. A concomitant rise in intracellular calcium to $0.51 \mu \mathrm{M}$ causes the
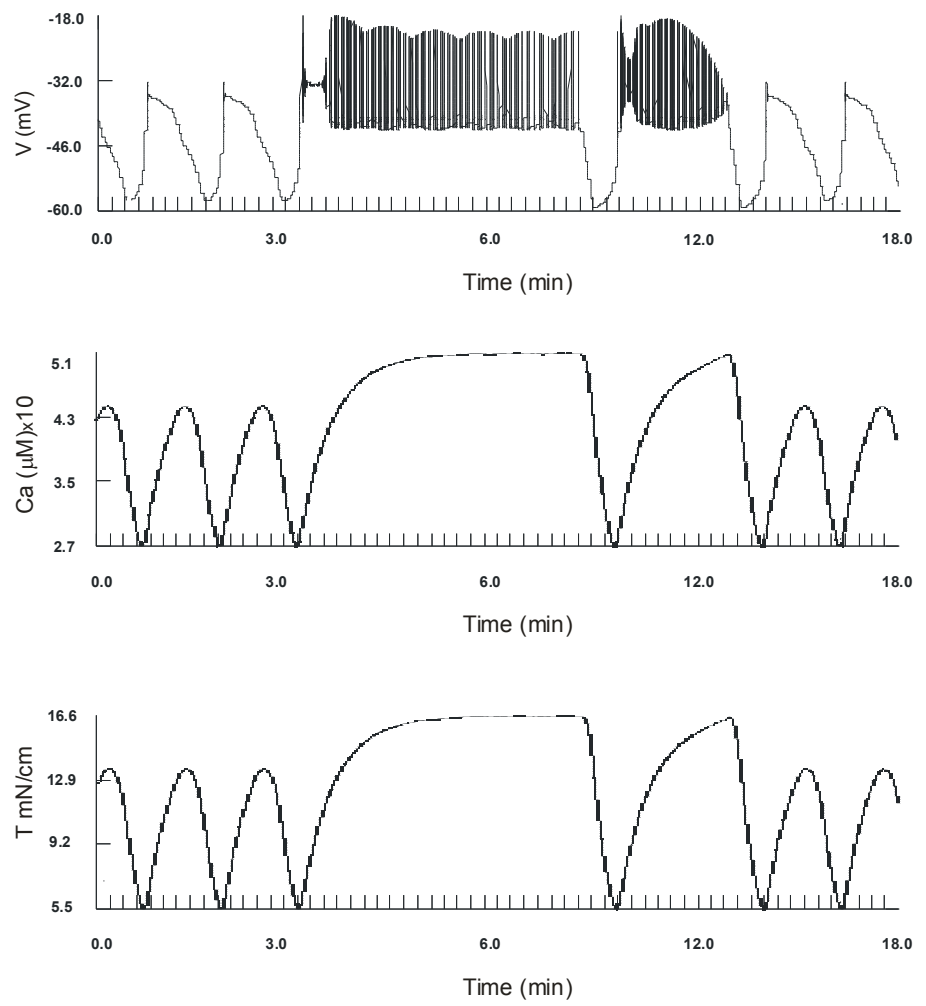

Figure 1: Dynamics of changes of the ion currents, membrane potential, cytosolic calcium and total force in the pregnant fasciculus at rest and during excitation. 

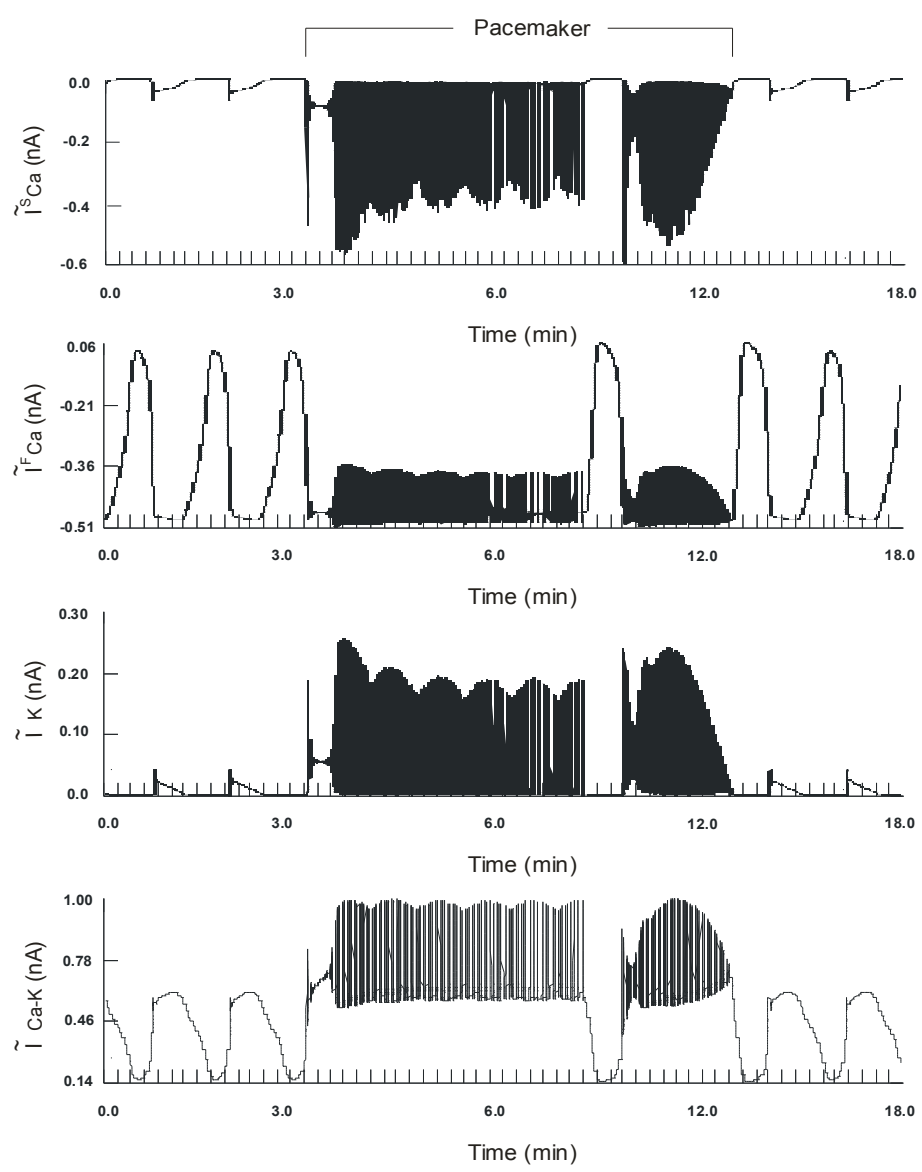

Figure 1: (Continued).

development of active force, $16.6 \mathrm{mN} / \mathrm{cm}$. Upon the termination of electrical discharges the myofiber returns to its unexcited state.

\subsection{Changes in $\mathrm{Ca}_{0}^{2+}$}

A gradual increase in the extracellular calcium leads to depolarization of the membrane. Concentrations of $\mathrm{Ca}_{0}^{2+} 3-5$ times normal causes the up-shift of the resting potential to $V^{r}=-34 \mathrm{mV}$ and $-31 \mathrm{mV}$, respectively. This is associated with the exponential rise in free cytosolic calcium to $0.72 \mu \mathrm{M}$. The myometrium undergoes tonic contraction, $T^{a}=23.75 \mathrm{mN} / \mathrm{cm}$. 
A concurrent electrical stimulation of the myofiber evokes ion currents, $\tilde{I}_{C a}^{s}=1.3 ; \tilde{I}_{C a}^{f}=0.61 ; \tilde{I}_{K 1}=0.62, \tilde{I}_{C a-K}=1.63, \tilde{I}_{C l}=1.89(\mathrm{nA})$, and a transient production of a burst of high frequency, $v=6 \mathrm{~Hz}$, action potentials of amplitude $53 \mathrm{mV}$. The $\mathrm{T}-\mathrm{Ca}^{2+}$ current provides the main influx of intracellular calcium during which a maximum of $0.62 \mu \mathrm{M}$ is recorded. The fasciculus generates the active force, $T^{a}=20.7 \mathrm{mN} / \mathrm{cm}$. The reversal of $\mathrm{Ca}_{0}^{2+}$ to its physiological level brings the myofiber to its original electromechanical activity.

Slow wave oscillations cease in a calcium free environment. The fasciculus becomes hyperpolarized at the constant level, $V=-50 \mathrm{mV}$. The concentration of intracellular calcium decreases to $0.1 \mu \mathrm{M}$ and is insufficient to sustain mechanical contractions. The myofiber remains relaxed.

\subsection{Changes in $\mathrm{K}_{0}^{+}$}

A two-fold increase in the concentration of extracellular potassium depolarizes the membrane, $V^{r}=-30 \mathrm{mV}$, and abolishes slow waves. The ion currents display a constant dynamics: $\tilde{I}_{C a}^{s}=0.04 ; \quad \tilde{I}_{C a}^{f}=0.48 ; \quad \tilde{I}_{K 1}=0.42 ; \quad \tilde{I}_{C a-K}=0.51$ (nA). $\mathrm{L}$ - and $\mathrm{T}-\mathrm{Ca}^{2+}$ currents contribute equally to the rise in $\left[\mathrm{Ca}^{2+}\right]$ to $0.5 \mu \mathrm{M}$ and the contraction of the myometrium, $T^{a}=15 \mathrm{mN} / \mathrm{cm}$.

The following four-fold increase in $\mathrm{K}_{0}^{+}$further depolarizes the membrane, $V^{r}=-20 \mathrm{mV}$. The intensity of the $K^{+}$current increases to $0.52 \mathrm{nA}$ with a concomitant attenuation of the respective currents: $\tilde{I}_{C a}^{s}=0.032 ; \tilde{I}_{C a}^{f}=0.39$ and $\tilde{I}_{C a-K}=0.14(\mathrm{nA})$. There is an exponential decline in $\left[\mathrm{Ca}^{2+}\right]$ to $0.44 \mu \mathrm{M}$ and in the intensity of force, $T^{a}=13.6 \mathrm{mN} / \mathrm{cm}$.

A superimposed electrical excitation leads to a burst of high amplitude, $V=$ $30 \mathrm{mV}$, and frequency, $v=7.3 \mathrm{~Hz}$, action potentials. The intracellular calcium content rises to $0.52 \mu \mathrm{M}$, and the fasciculus produces the active force of 16.8 $\mathrm{mN} / \mathrm{cm}$.

A simultaneous elevation in $\mathrm{K}_{0}^{+}$and $\mathrm{Ca}_{0}^{2+}$ stabilizes the membrane potential at $-18 \mathrm{mV}$. The intracellular $\left[\mathrm{Ca}^{2+}\right]=0.65 \mu \mathrm{M}$ triggers a strong contraction of the myofiber, $\max T^{a}=21.8 \mathrm{mN} / \mathrm{cm}$.

A gradual reduction of $\left[\mathrm{K}_{0}^{+}\right]$hyperpolarizes the fasciculus, $V^{r}=-70$ and $88(\mathrm{mV})$. The slow wave amplitude and frequency increase to $40 \mathrm{mV}, v=0.032$ $\mathrm{Hz}$ and $58 \mathrm{mV}, v=0.037 \mathrm{~Hz}$. Concurrent multiple discharges of a pacemaker evoke the production of spikes of an average amplitude of $60 \mathrm{mV}$ at a frequency of $\sim 6 \mathrm{~Hz}$. There is a weakening of the calcium influx, $\max \left[\mathrm{Ca}^{2+}\right]=0.25$ and 
$0.18(\mu \mathrm{M})$, and tension, $T^{a}=4.5$ and $2.2(\mathrm{mN} / \mathrm{cm})$. Interestingly, the electrical stimulation further reduces the strength of contraction, $T^{a}=1.1(\mathrm{mN} / \mathrm{cm})$. The duration of contractions also decreases.

\section{Conclusion}

The knowledge of electromechanical behaviour of the myometrium is crucial for the integration of motor functions into a biologically plausible biomechanical model of the pregnant uterus. However, because of the paucity of experimental data it is impossible to provide a comprehensive quantitative analysis of the validity, accuracy and applicability of the above results. Qualitative comparison to in vivo and in vitro recordings of electromechanical activity of the pregnant human myometrial tissue demonstrates a satisfactory correlation with the numerical results.

\section{References}

[1] Vauge, C., Carbonne, B., Papiernik, E. and Ferré, F. (2000). A mathematical model of uterine dynamics and its application to human parturition. Acta Biotheoretica, 48, pp. 95-105, 2000

[2] Bursztyn, L., Eytan, O., Jaffa, A.J. \& Elad, D. Mathematical model of excitation-contraction in a uterine smooth muscle cell. American Journal of Physiology, Cell Physiology 292, pp. C1816-C1829, 2007

[3] Young, R. \& Hession, R. Three-dimensional structure of the smooth muscle in the term-pregnant human uterus. Obstetrics \& Gynecology, 93(1), pp. 9499, 1999

[4] Garfield R. E. \& Maner, W. L. Physiology of electrical activity of uterine contractions. Seminars on Cell Developmental Biology, 18(3) pp. 289-295, 2007 